\title{
Analyzing the Impact of Trucks on Traffic Flow Based on an Improved Cellular Automaton Model
}

\author{
Dewen Kong, ${ }^{1,2,3}$ Xiucheng Guo, ${ }^{3}$ Bo Yang, ${ }^{4}$ and Dingxin $\mathrm{Wu}^{3}$ \\ ${ }^{1}$ Jiangsu Key Laboratory of Urban ITS, Southeast University, Nanjing 210096, China \\ ${ }^{2}$ Jiangsu Province Collaborative Innovation Center of Modern Urban Traffic Technologies, Southeast University, Nanjing 210096, China \\ ${ }^{3}$ School of Transportation, Southeast University, Nanjing 210096, China \\ ${ }^{4}$ Department of Civil, Construction and Environmental Engineering, North Carolina State University, Raleigh, NC 27695, USA
}

Correspondence should be addressed to Xiucheng Guo; seubluesky@126.com

Received 14 June 2016; Revised 15 August 2016; Accepted 30 August 2016

Academic Editor: Ricardo López-Ruiz

Copyright (c) 2016 Dewen Kong et al. This is an open access article distributed under the Creative Commons Attribution License, which permits unrestricted use, distribution, and reproduction in any medium, provided the original work is properly cited.

\begin{abstract}
This paper aims to analyze the impact of trucks on traffic flow and propose an improved cellular automaton model, which considers both the performance difference between passenger cars and trucks and the behaviour change of passenger cars under the impact of trucks. A questionnaire survey has been conducted to find out whether the impact of trucks exists and how the behaviour of passenger car drivers changes under the impact of trucks. The survey results confirm that the impact of trucks exists and indicate that passenger car drivers will enlarge the space gap, decelerate, and change lanes in advance when they are affected. Simulation results show that traffic volume is still affected by percentages of trucks in the congestion phase in the proposed model compared with traditional heterogeneous cellular automaton models. Traffic volume and speed decrease with the impact of trucks in the congestion phase. The impact of trucks can increase traffic congestion as it increases. However, it has different influences on the speed variance of passenger cars in different occupancies. In the proposed model, the relative relationship of the space gap between car-following-truck and car-following-car is changeable at a certain value of occupancy, which is related to the impact of trucks.
\end{abstract}

\section{Introduction}

With the development of truck transportation, the number of trucks and their proportion in freeway traffic have been increasing significantly in the world. The average percentage of trucks in most freeways in China is around $25 \%$ and some can even reach as much as $60 \%$ [1]. The number of trucks has increased by $75 \%$ in the USA and this trend will continue over the next decade [2]. The development tendency of trucks in Australia is consistent and the kilometers travelled by trucks are predicted to increase by $74 \%$ between 2003 and 2020 [3]. Although trucks play a key role in road freight transport, they are also an important factor of traffic crash and congestion. Trucks have a great impact on freeway capacity and freeway overall performance, among others, because of their physical (e.g., size) and operational (e.g., acceleration/deceleration) characteristics [4]. Large-sized trucks can block the sight distance of following vehicles and trucks will obstruct the operations of fast vehicles due to the poor acceleration and maximum speed. In addition, it has been found that trucks have an influence on surrounding vehicles, which makes the driving behaviour of these vehicles change [5]. In general, although trucks only occupy a small proportion of traffic flow, they have a pronounced impact on the traffic. Hence, it is important to study the impact of trucks to help improve traffic operations.

The study of traffic flow has attracted many researchers from different fields because of its complexity and comprehensiveness and many theoretical approaches have been presented, such as hydrodynamic models, gas kinetic models, car-following models, and cellular automaton (CA) models $[6,7]$. Among them, a CA model is a microscopic model used to construct complex systems and the time, space, and state of it are all discrete. It can use fairly simple rules to reflect complicated vehicle behaviour and interaction in the real world and has a high computational efficiency. Another 
advantage of a CA model is that it is easy for computer simulation because there is no transformation of "discretecontinuous-discrete." Therefore, a CA model is widely used to simulate vehicle movements on the road. Di Gregorio and Festa, Cremer, and Ludwig proposed the CA traffic model earlier $[8,9]$. Afterwards, Nagel and Schreckenberg proposed the NaSch model, the most famous CA models, in 1992, thus extending the maximum speed and introducing the randomization probability [10]. The NaSch model has laid a basis for the development of CA models later. Many CA models have been developed based on the NaSch model to reflect some real and special phenomena in the world, such as metastable situations and oscillatory congested states $[11,12]$. These models include the FI model that improved the acceleration rules [13], the VDR model that introduced the slow-to-start rules [14], and the BL model that considered the effect of preceding vehicles [15]. Since most roads in the world have two or more lanes in one direction, lane-change rules have been developed from the single-lane CA model into the multilane CA model. Some complex traffic problems have been simulated through these models and abundant research results have been obtained [16-18].

Heterogeneous traffic flow composed of passenger cars and trucks is a common phenomenon in road traffic. However, few CA models are built for heterogeneous traffic flow. Most of them are built for homogenous traffic flow currently, which means only cars in the system. Meng and Weng have constructed a heterogeneous CA model for freeway work zones. There were two vehicle types in the system and each had different characteristics [19]. Jia et al., Ebersbach et al., and Xiao et al. used the heterogeneous CA model to study the impact of trucks on traffic flow. They studied the influence of vehicle length, truck percentage, and maximum speed on the system performance [20-22]. For the existing CA models, when modelling heterogeneous traffic flow, they only give different characteristic parameters (e.g., vehicle length, acceleration, and maximum speed) for different vehicle types, but the update rules are still the same. In this case, no difference of the behaviour of passenger cars can be observed when under the influences of trucks and cars. Therefore, the impact of trucks on traffic flow as reflected by these CA models is determined mainly from the differences of performance between passenger cars and trucks, while the impact of trucks on the behaviour of passenger cars is ignored in these models. However, many studies have proved that passenger cars are frequently affected by trucks on the road and their driving behaviour will change significantly, such as headway, speed choice, and lane-change decision [23]. Therefore, the existing heterogeneous CA models will underestimate the impact of trucks on traffic flow and some simulation results may be far from the actual traffic flow. The heterogeneous CA models could be improved dramatically by considering the impact of trucks.

The primary objective of this study is to analyze the impact of trucks on traffic flow using an improved heterogeneous CA model. More specifically, this study includes the following two tasks: (1) to propose an improved heterogeneous CA model considering both the performance differences between passenger cars and trucks and the impact of trucks on the behaviour of passenger cars and (2) to analyze the impact of trucks on traffic flow under different conditions through computer simulation. In the following section, a survey is performed regarding the impact of trucks. In Section 3, the development of the improved heterogeneous CA model is presented. Section 4 shows the simulation results and analysis. The findings are summarized while conclusions and suggestions for further research are provided in the end.

\section{Survey and Data Analysis}

In order to understand the impact of trucks on the behaviour of passenger car drivers, a questionnaire survey has been conducted in Nanjing, Jiangsu Province, China. The respondents were passenger car drivers. The survey was conducted in three places. The first survey was conducted in a service area of Shanghai-Nanjing freeway on December 1, 2015. A total of 165 responses from passenger car drivers were collected. The second survey was conducted in the parking lot of Nanjing Railway Station on December 6, 2015. A total of 200 responses were collected. The third survey was conducted in the parking lot of a supermarket on December 12, 2015. A total of 185 responses were collected. After removing the invalid questionnaires, 520 samples were obtained. Among the samples, in regard to the gender, $31.73 \%$ were females and $68.27 \%$ were males. Concerning their age, $54.81 \%$ were under 30 years of age; $15.38 \%$ were between 30 and 40 years of age; $27.88 \%$ were between 40 and 50 years of age; and only $1.92 \%$ were above 50 years of age. For their driving experience, $38.46 \%$ had less than 1 year of driving experience, $29.81 \%$ had from 1 to 5 years, and $31.73 \%$ had more than 5 years.

There are 5 questions in this survey and the results are presented in Figure 1. The first is whether there is an impact when passenger car drivers drive behind a truck. The results displayed that $95.77 \%$ of the passenger car drivers indicated that the impact existed. A 7-point Likert scale was used to let these drivers estimate the degree of the impact, where 1 denoted very mild and 7 very serious. The minimum value obtained was 2 and the maximum value was 7 . The average degree of the impact was 4.34 and variation was 2.09 . These results mean that trucks have a significant impact on the following passenger cars. The second question is what the biggest impacts of trucks are. The three main truck impacts were sight distance obstruction, speed reduction, and psychological pressure in proportions of $39.42 \%, 34.62 \%$, and $17.31 \%$, respectively. It can be found that the big size and poor operation performance result in the impact of trucks and psychological pressure cannot be ignored. The third and fourth questions are about the car-following behaviour when following a truck. Almost all the respondents chose to enlarge the space gap when following a truck (89.42\%), only $2.89 \%$ chose to narrow the space gap, and $7.69 \%$ chose to keep the constant space gap. The speed choice was investigated in the fourth question. $72.12 \%$ of the drivers would decelerate and $27.88 \%$ of the drivers would still keep the same speed when they felt the impact of a truck. From these two questions, it is obvious that passenger car drivers intend to keep a 

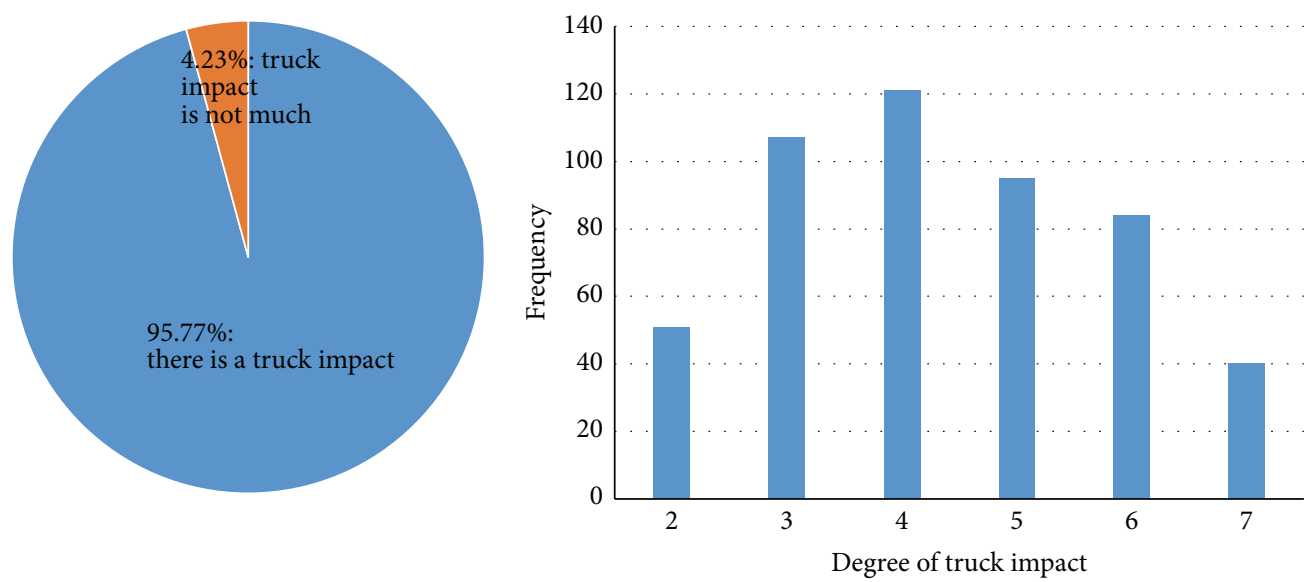

(a) The results of question one

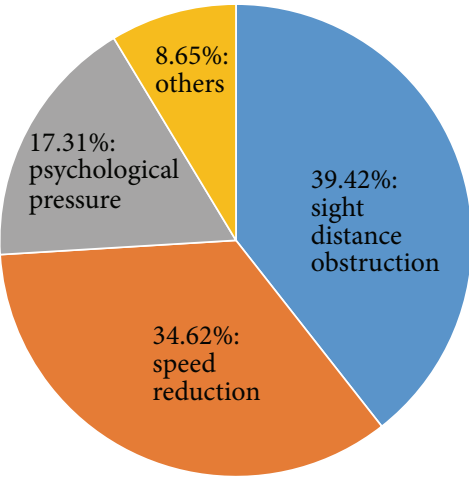

(b) The results of question two
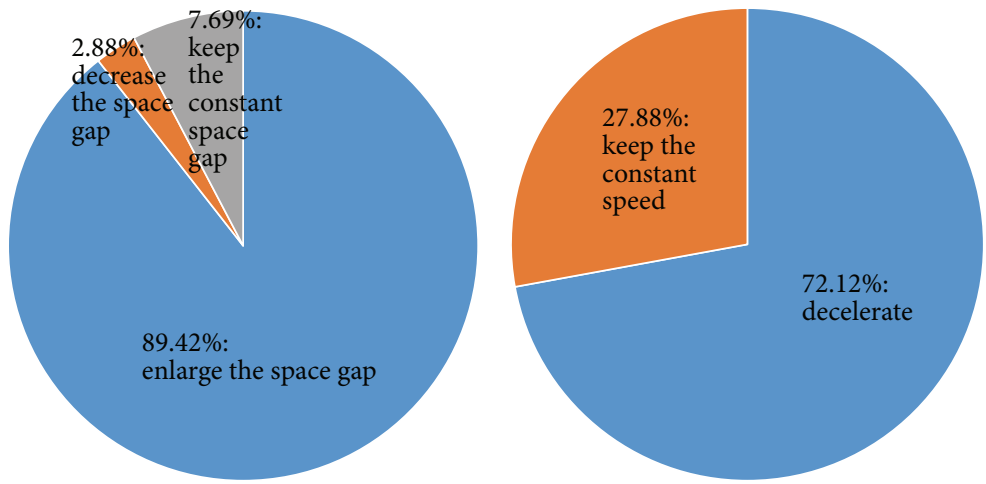

(c) The results of questions three and four

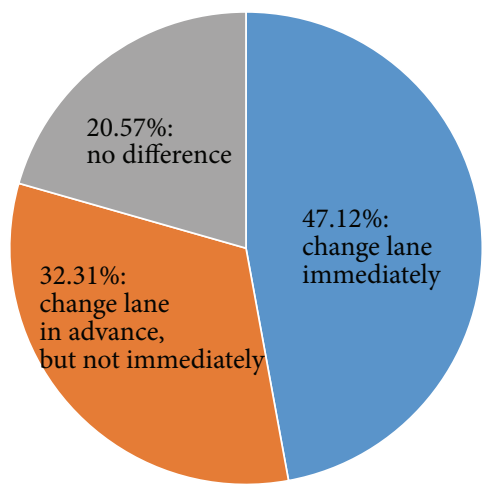

(d) The results of question five

FIgURE 1: The results of the questionnaire survey.

larger distance between the preceding truck and themselves and decelerate. The last question is about the lane-change behaviour when following a truck. $47.12 \%$ of the drivers chose to change lanes immediately when they felt the impact of a truck. $32.31 \%$ of the drivers chose to change lanes in advance, but not immediately. $20.57 \%$ of the drivers' lane-change behaviour was just the same as that when following a car. Moreover, a further question was concerned with whether they would change lanes in advance when following a truck even though the current lane can still satisfy their speed. $54.48 \%$ of the drivers said yes. The fifth question indicates that the impact of trucks makes passenger car drivers tend to change lanes.

From the survey, the impact of trucks on the following passenger car exists, including sight distance obstruction, speed reduction, and psychological pressure. The behaviour of passenger car drivers will change under the impact, like to enlarge the space gap, decelerate, and change lanes in advance. Therefore, if the update rules in the CA model are still the same for passenger cars under the impact of trucks, it would cause some problems and reduce the accuracy of the model. 


\section{Improved CA Model and Relevant Parameters}

In this section, the parameters used in the CA model are first presented. Then the improved CA model is proposed to consider the impact of trucks.

3.1. Basic Parameters. The simulated road consists of two lanes: lane 0 and lane 1 . Each lane is divided into $L$ cells. At every time step, the cell is empty or occupied by a vehicle. There are two vehicle types, passenger cars and trucks, in the system.

$V_{n}^{i}(t)$ represents the speed of vehicle $n$ on lane $i$ at time $t$, which is an integer value among $0,1,2,3, \ldots, V_{\max } . V_{\max }$ is the maximum speed. $x_{n}^{i}(t)$ denotes the position of vehicle $n$ on lane $i$ at time $t$. The space gap between vehicle $n$ and its preceding vehicle $n+1$ at time $t$ is $d_{n}^{i}(t)=x_{n+1}^{i}(t)-$ $x_{n}^{i}(t)-l_{n+1} \cdot l_{n+1}$ represents the length of vehicle $n+1$. $d_{n \text { front }}^{\prime}(t)$ and $d_{n \text { back }}^{\prime}(t)$ represent the space gap between vehicle $n$ and its two neighbour vehicles on the other lane. The acceleration and deceleration rates are represented by acc and dec, respectively. The time step is represented by $T$. In the improved CA model, imp is introduced to indicate the impact of trucks quantitatively. The impact of trucks increases with the growth of imp. Actually, imp is a very complicated parameter because it is variable in different conditions and sensitive to lots of factors, such as the type of trucks, the car driver's characteristics, and the relative relationship between the car and its front truck. Moreover, the relationship between imp and these factors is complex. Since we do not know how these factors affect imp specifically because of the lack of the data for this aspect, we made this problem simplified that trucks will produce the same impact when cars are following the trucks; that is, the values of imp for all the trucks are identical. This simplification makes the simulation in the experiment more feasible and easier. Also, by this way, it is more convenient to study the impact of trucks on traffic flow by changing the value of imp.

3.2. Improved CA Model. When simulating vehicle movements on the road, the update rules for the passenger car following a truck are different. We call it the truck impact rule. In other cases, (e.g., the passenger car following a car) the basic rule is used, which is based on the NaSch model.

3.2.1. Lane-Change Rules. In the basic rule, vehicles will change lanes with probability $p_{l}$ when the following three criterions are satisfied.

Interval criterion:

$$
t_{n} \geq t_{h}
$$

Incentive criterion:

$$
\begin{array}{r}
\min \left(V_{n}^{i}(t)+\operatorname{acc} * T, V_{\max }\right) * T>d_{n}^{i}(t), \\
d_{n \text { front }}^{\prime}>d_{n}^{i}(t) .
\end{array}
$$

Security criterion:

$$
\begin{aligned}
d_{n \text { back }}^{\prime}(t) \geq & \min \left(V_{b}(t)+\operatorname{acc} * T, V_{\max }\right) * T \\
& -\min \left(V_{n}^{i}(t)+\operatorname{acc} * T, V_{\max }\right) * T+\text { saf. }
\end{aligned}
$$

$p_{l}$ is the critical lane-change probability. $t_{n}$ and $t_{h}$, respectively, represent the time interval of lane change of vehicle $n$ and the permitted time interval of lane change, which are used to avoid changing lanes frequently. $V_{b}(t)$ is the speed of the neighbour following vehicle on the other lane at time $t$. saf denotes the buffer distance. The incentive criterion means vehicle $n$ cannot drive at desired speed on the current lane and the condition of the other lane is better than the current lane. The security criterion used here considers the relative motion of vehicle $n$ and its neighbour following vehicle.

Based on the survey, when a passenger car follows a truck, the passenger car driver will change lanes in advance even though the condition of the current lane can still satisfy his/her speed. Therefore, the incentive criterion in the basic rule is improved to be used in the truck impact rule. Other criterions in the basic rule and the truck impact rule are the same. The incentive criterion in the truck impact rule is as follows.

Incentive criterion:

$$
\begin{aligned}
& \min \left(V_{n}^{i}(t)+\operatorname{acc} * T, V_{\max }\right) * T> d_{n}^{i}(t), \\
& d_{n \text { front }}^{\prime}> d_{n}^{i}(t), \\
& \text { when } d_{n}^{i}(t) \geq \text { dis, } \\
& \min \left(V_{n}^{i}(t)+\operatorname{acc} * T, V_{\max }\right) * T> \frac{d_{n}^{i}(t)}{\operatorname{imp}+1}, \\
& d_{n f r o n t}^{\prime}> d_{n}^{i}(t), \\
& \text { when } d_{n}^{i}(t)<\text { dis, }
\end{aligned}
$$

where dis represents the influence distance of trucks and is assumed as a constant in this paper. The truck impact works if $d_{n}^{i}(t)$ is smaller than dis. When the passenger car is under the truck impact $\left(d_{n}^{i}(t)<\right.$ dis $)$, the driver will decide to change lanes in advance. Therefore, the threshold of lane change decreases $\left(d_{n}^{i}(t) /(\mathrm{imp}+1)\right.$ is smaller than $d_{n}^{i}(t)$ obviously when imp $>0)$. From $(4), d_{n}^{i}(t) /(\mathrm{imp}+1)$ decreases as imp increases, which means the incentive criterion is much easier to meet with larger imp. So the greater the impact of trucks is, the stronger the motivation of changing lanes is.

3.2.2. Car-Following Rules. In the car-following rules, vehicles will update their speed and longitudinal position. The four steps of car-following in the basic rule are as follows.

Acceleration:

$$
V_{n}^{i}(t+T) \longleftarrow \min \left(V_{n}^{i}(t)+\operatorname{acc} * T, V_{\max }\right) .
$$


Collision avoidance:

$$
\begin{aligned}
& V_{n}^{i}(t+T) \\
& \longleftarrow \min \left(V_{n}^{i}(t+T), \frac{\left(d_{n}^{i}(t)+\lambda * V_{n+1}^{i^{\prime}}(t) * T\right)}{T}\right) .
\end{aligned}
$$

Randomization:

$$
\begin{array}{r}
V_{n}^{i}(t+T) \longleftarrow \max \left(V_{n}^{i}(t+T)-\operatorname{dec} * T, 0\right) \\
\text { with probability } p .
\end{array}
$$

Movement:

$$
x_{n}^{i}(t+T) \longleftarrow x_{n}^{i}(t)+V_{n}^{i}(t+T) * T .
$$

In collision avoidance, $V_{n+1}^{i \prime}(t)=\max \left(\min \left(V_{n+1}^{i}(t), d_{n+1}^{i}(t) /\right.\right.$ $T)-\operatorname{dec} * T, 0)$ is the predicted speed of the preceding vehicle. $\lambda$ is the anticipated parameter that reflects the degree of aggressive driving. $p$ is the randomization probability.

Based on the survey, when a passenger car follows a truck, the passenger car driver will enlarge the space gap and decelerate. Hence, the steps of collision avoidance and randomization are modified to be used in the truck impact rule. Other steps in the truck impact rule are the same as those in the basic rule. The modified steps of collision avoidance and randomization in the truck impact rule are as follows.

Collision avoidance:

$$
\begin{aligned}
& V_{n}^{i}(t+T) \longleftarrow \min \left(V_{n}^{i}(t+T),\right. \\
& \left.\quad \frac{\left(d_{n}^{i}(t)+\lambda /(\operatorname{imp}+1) * V_{n+1}^{i^{\prime}}(t) * T\right)}{T}\right) .
\end{aligned}
$$

Randomization:

$$
\begin{aligned}
& V_{n}^{i}(t+T) \longleftarrow \max \left(V_{n}^{i}(t+T)-\operatorname{dec} * T, 0\right) \\
& \text { with probability } p \text {, when } d_{n}^{i}(t) \geq \text { dis, } \\
& V_{n}^{i}(t+T) \longleftarrow \max \left(V_{n}^{i}(t+T)-\operatorname{dec} * T, 0\right) \\
& \text { with probability } p+\left(1-\frac{d_{n}^{i}(t)}{\operatorname{dis}}\right) * a * \text { imp, when } d_{n}^{i}(t)<\text { dis. }
\end{aligned}
$$

Because of the impact of trucks, the sight distance of the following car is obstructed. Hence, in collision avoidance, the driver will anticipate the speed of the preceding truck conservatively to enlarge the space gap. The passenger car driver will drive more safely with an increase in the impacts of trucks. When the passenger car suffers the impact of trucks, the driver will also drive very carefully and control the speed all the time. Therefore, the probability of deceleration increases, signifying that the randomization probability increases. The randomization probability is related to the space gap and the impact of trucks. The greater the impact of trucks is and the closer the space gap is, the higher the randomization probability is. $a$ is a fixed constant.

\section{Numerical Simulation and Analysis}

Most previous CA models set the cell size with $7.5 \mathrm{~m}$, which is too coarse, leading to unrealistic acceleration and deceleration rates. In the improved CA model, according to the actual sizes of vehicles, acceleration and deceleration rates, and so forth, in the used data set and the computational speed, the cell size is set as $1.5 \mathrm{~m}$. A numerical simulation was performed with periodic boundary conditions and the road length $L$ is 5000 cells, that is, $7.5 \mathrm{~km}$. For passenger cars, $V_{\max }$ was set as 25 cells $/ \mathrm{s}(135 \mathrm{~km} / \mathrm{h})$ and acc and $\mathrm{dec}$ were taken as 2 cells $/ \mathrm{s}^{2}\left(3 \mathrm{~m} / \mathrm{s}^{2}\right)$, while $l$ was 5 cells $(7.5 \mathrm{~m})$. For trucks, $V_{\max }$ was set as 15 cells $/ \mathrm{s}(81 \mathrm{~km} / \mathrm{h})$ and acc and dec were taken as 1 cell $/ \mathrm{s}^{2}\left(1.5 \mathrm{~m} / \mathrm{s}^{2}\right)$, while $l$ was 10 cells $(15 \mathrm{~m})$. These parameters refer to the trajectory data collected at southbound direction of the US highway 101 in Los Angeles, California, from the Next Generation Simulation project. The anticipated parameter $\lambda$, randomization probability $p$, and critical lane-change probability $p_{1}$ were $0.5,0.2$, and 0.5 , respectively. The permitted time interval of lane change $t_{h}$ was $4 \mathrm{~s}$, while buffer distance saf was set as 2 cells $(3 \mathrm{~m})$ [16]. The fixed constant $a$ was taken as 0.08 , which is used to guarantee $p+\left(1-d_{n}^{i}(t) /\right.$ dis $) * a * \operatorname{imp} \leq 1$. In addition, the influence distance of trucks dis was taken as 50 cells $(75 \mathrm{~m})$ according to the literature [24].

Note that the effect and magnitude are two factors in the study of the impact of trucks. In this study, however, the magnitude of the impact of trucks was not determined in detail through the survey. The emphasis of our study is to introduce the impact of trucks to the CA model and analyze the feature of traffic flow by changing it. For example, the fundamental diagram is determined by different imp and the effect is studied. Hence, it is not necessary to determine the absolute magnitude of the impact of trucks in this study. In some places of this study, the magnitude of the impact of trucks (i.e., imp) needs to be fixed to study the effect of the percentage of trucks. In this case, the value of imp will be set as 6 based on the result of question one in the survey $(4.34 / 7 * 10 \approx 6$, since the range of the impact of trucks in the CA model is 0 to 10$)$.

Let the total number of vehicles on the road be $N$; then the vehicle density was $\rho=N / 2 L$. If the percentage of trucks was $r$, then the occupancy would be $C=\left(N * r * l_{t}+N *(1-\right.$ $\left.r) * l_{c}\right) / 2 L$. The relationship between density and occupancy met $\rho=C /\left(r * l_{t}+(1-r) * l_{c}\right)$. The average system speed at time $t$ was $V(t)=(1 / N) \sum_{i=0}^{1} \sum_{n=1}^{N^{i}} V_{n}^{i}(t)$, where $N^{i}$ was the number of vehicles on lane $i$. Hence, traffic volume at time $i$ was $Q=\rho * V(t)$.

At the initial time, passenger cars and trucks were randomly distributed on the road according to the percentage of trucks, and the initial speed of each vehicle was a random value. Each time step $T$ corresponds to 1 second. The system simulated 20000 time steps each time. To exclude the transient time, only the traffic parameters of final 2000 time steps were collected and analyzed. Meanwhile, to eliminate the impact of randomness, 20 samples were averaged. By changing $C, r$, and imp, traffic was simulated under different conditions. 


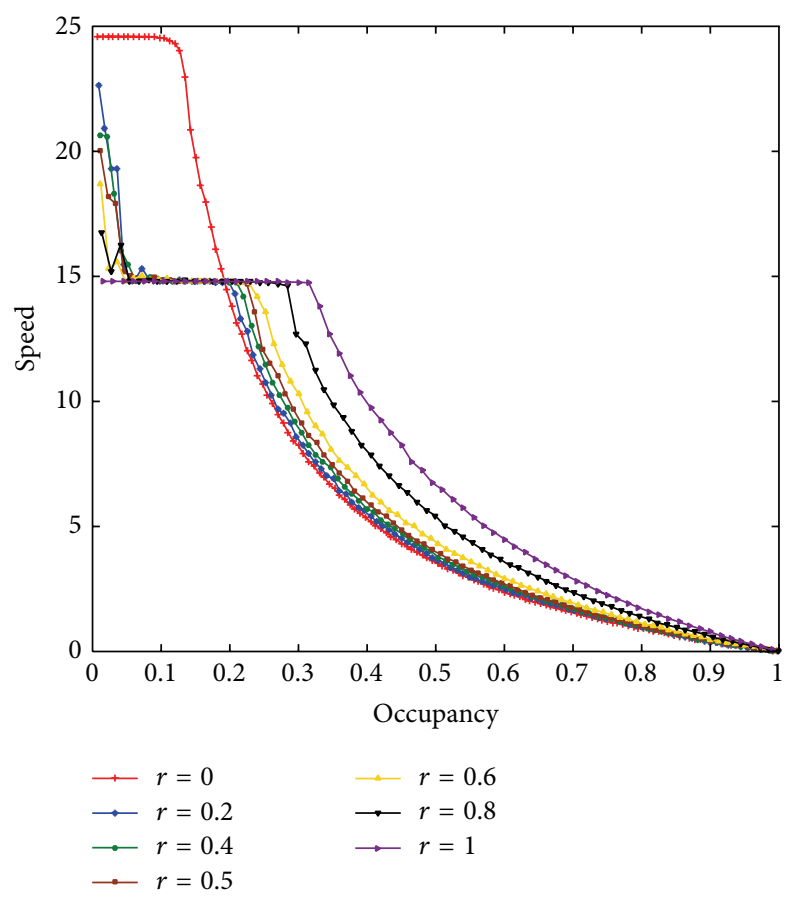

(a) Speed versus occupancy

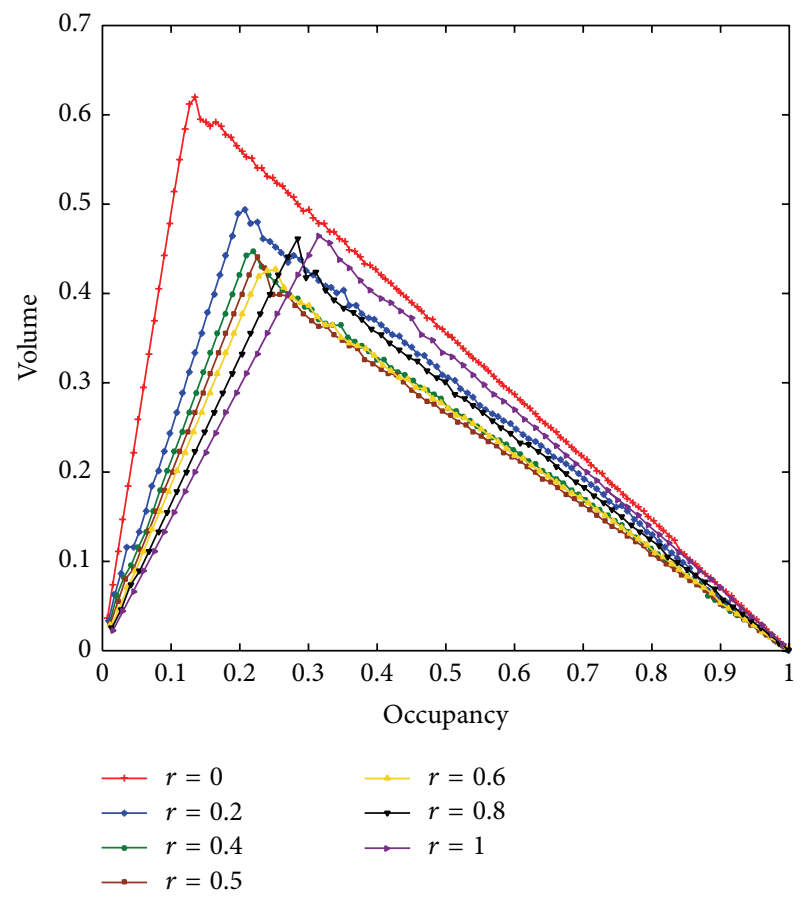

(b) Volume versus occupancy

Figure 2: The fundamental diagram under different $r$ when imp $=6$.

4.1. Analysis of Fundamental Diagram. The relationship between speed and occupancy with different percentages of trucks when imp $=6$ is presented in Figure 2(a). The speed is divided into two regions when the traffic is homogenous (only passenger cars or trucks in the system). In the region $C<C_{\text {critical }}$, the speed remains unchanged and meets the relationship $\bar{V}=V_{\max }-p$. In the region $C>C_{\text {critical }}$, the speed decreases with the occupancy. The speed is classified into three regions when the traffic is heterogeneous (both passenger cars and trucks in the system). The speed decreases sharply to the vicinity of the maximum speed of trucks in the region $C<0.05$. The speed tendency in the regions $0.05<C<C_{\text {critical }}$ and $C>C_{\text {critical }}$ is similar to that in the homogenous traffic described above. These results indicate that passenger cars are affected by trucks significantly and cannot maintain their own speed. In the congestion phase, the speed of heterogeneous traffic is between that of the passenger car-only traffic and that of the truck-only traffic and increases with the percentage of trucks. Since a truck can occupy more cells than a car, under the same occupancy, the total number of vehicles decreases as the percentage of trucks increases. Therefore, the interaction between vehicles is weakened in the congestion phase and the average speed of traffic flow increases. Figure 2(b) shows the relationship between traffic volume and occupancy with different percentages of trucks when imp $=6$. Traffic volume increases with the occupancy when $C<C_{\text {critical }}$ and decreases with the occupancy when $C>C_{\text {critical }}$. With an increase in percentages of trucks, the critical occupancy $C_{\text {critical }}$ also increases. The value $C_{\text {critical }}$ increases from 0.135 at $r=0$ to 0.315 at $r=$ 1. A significant difference can be found by comparing the relationship between traffic volume and occupancy in the proposed model with that in the model advocated in the literatures $[20,22,25]$ (see Figure 3(b)), which only have different characteristic parameters for different vehicle types (i.e., the proposed model when imp $=0$ ). In Figure $3(b)$, the maximum traffic volume decreases with the percentages of trucks. When $C>C_{\text {critical }}$, traffic volume curves at different percentages of trucks gather together, which indicates that traffic volume is not influenced by the percentages of trucks. However, in Figure 2(b), the maximum traffic volume first decreases and then increases with the percentages of trucks and traffic volume curves at different percentages of trucks do not gather together when $C>C_{\text {critical }}$. In the region $C>C_{\text {critical }}$, traffic volume decreases from $r=0$ to $r=0.5$ and reaches the minimum value at $r=0.5$. Then it increases from $r=0.5$ to $r=1$, suggesting that the percentages of trucks still have an influence on traffic volume in the congestion phase and the higher the mixed degree is, the more greatly the traffic volume decreases. To explain this phenomenon, the relationship between speed and occupancy from the two CA models was compared, as shown in Figures 2(a) and 3(a). In free flow, the relationships between speed and occupancy in the two CA models stay the same. However, in the congestion phase, although the speed increases with the percentages of trucks, it increases little in the proposed CA model when $r \leq 0.6$. It is well known that volume equals speed multiplied by density. Under the same occupancy, the density decreases and speed increases with the percentages of trucks. In the CA models without considering the impact of trucks, the effect of decreased density counteracts the effect of increased speed and the volume keeps the same. Hence, it 


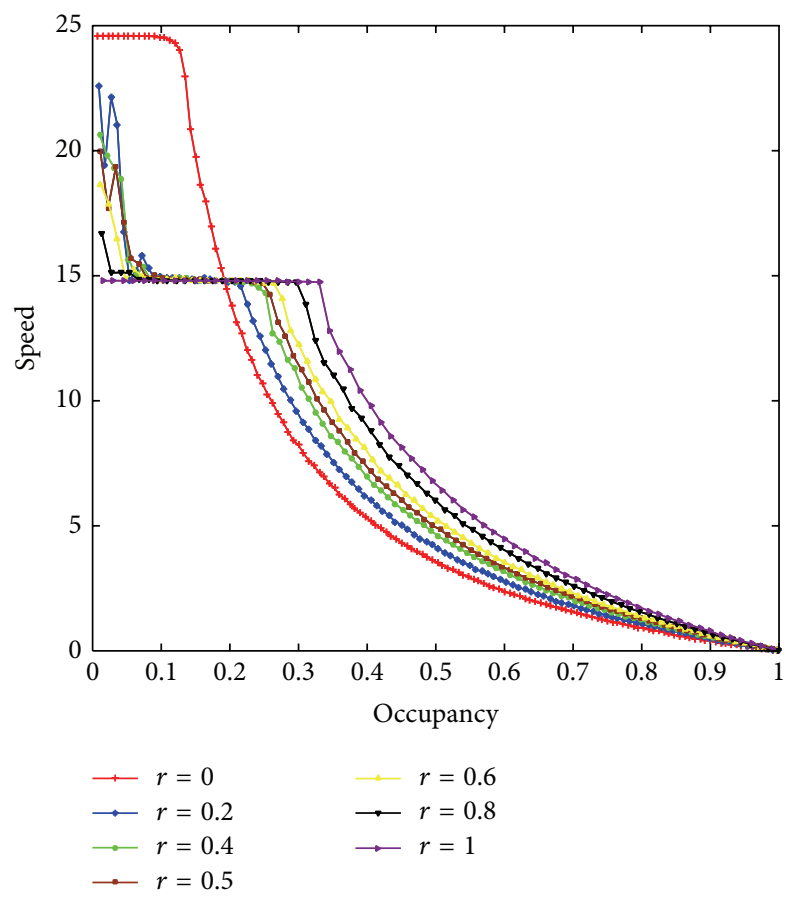

(a) Speed versus occupancy

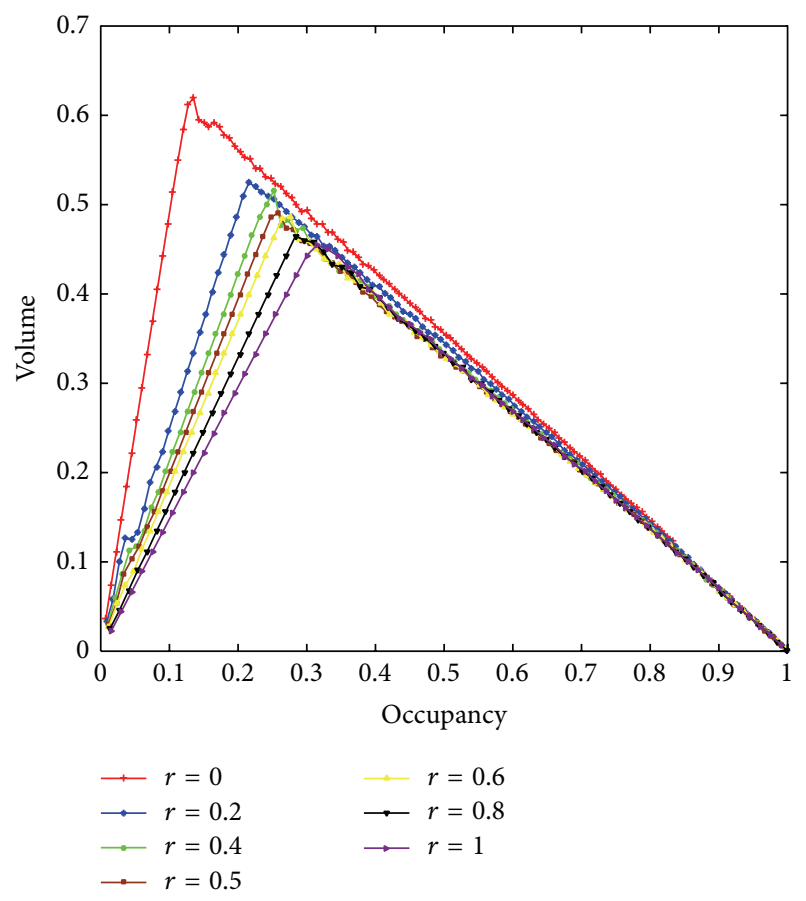

(b) Volume versus occupancy

Figure 3: The fundamental diagram under different $r$ when imp $=0$.

presents that the percentages of trucks have no influence on traffic volume. Nevertheless, in the proposed model, since the speed increases slowly when $r \leq 0.6$, the effect of decreased density is larger than the effect of increased speed and the volume decreases. After the speed increases fast when $r>$ 0.6 , the effect of increased speed increases and the volume becomes higher. Hence, the interesting phenomenon appears in the proposed model. The main reason for this difference is that the proposed model considers the behaviour change of passenger cars under the impact of trucks. Passenger cars, as a class of particles in the system, have high manoeuvrability and are easy to be influenced by the surrounding environment. When $r=0.4,0.5,0.6$, trucks are close in number to passenger cars, and the probability of passenger car following truck is much higher on the road. Passenger cars choose to decelerate and change lanes because of the frequent truck impact. When the occupancy is high, the lane change will disturb the following vehicles on the other road and deteriorate the system efficiency. When $r=0.2$ or $r=0.8$, either few passenger cars or few trucks, the disturbance to the system is limited and efficiency does not decrease too much. In the CA models without considering the impact of trucks, the available cells increase with the percentages of trucks and passenger cars will use these cells to accelerate as far as possible and do not care about the existence of trucks. That is unrealistic in the real world.

The fundamental diagram with different truck impacts at $r=0.2$ is presented in Figure 4. In free flow, traffic volume and speed are independent of the impact of trucks. However, the volume and speed both decrease with the impact of trucks in the congestion phase. It reflects that the impact of trucks works under congested traffic conditions. The critical occupancy $C_{\text {critical }}$ also becomes smaller because of the higher truck impact. When $C<C_{\text {critical }}$, the intervehicle interaction is weak, so the impact of trucks is insignificant. When $C>C_{\text {critical }}$, the number of passenger cars influenced by trucks increases, so the impact of trucks becomes significant. Passenger car drivers control the speed and enlarge the space gap, leading to reduced traffic volume and speed.

4.2. Analysis of Spatiotemporal Dynamics. For a deeper understanding of traffic flow dynamics under different conditions, the spatial-temporal diagrams were obtained by simulations as shown in Figure 5. In the figures, the spatial position range is $0-5000$, while the time step is 1950020000. The horizontal axis represents time and the vertical axis represents position. Black dots indicate the position of vehicles, while white dots indicate blank space. Figures 5(a)5(d) compare the system state at the truck impacts of 0 and 10 , respectively, when $r=0.2$. When the occupancy is 0.05 , the system is free flow and trucks have no marked impacts and vehicles are distributed evenly with straight tracks. When the occupancy is 0.225 , local congestion appears in both Figures 5(c) and 5(d), which propagates backward. A comparison of the two figures shows that when the impact of trucks is 0 , local congestion occurs and continues for only a short period of time and then disappears automatically. When the impact of trucks is 10, more local congestion instances appear and some do not disappear but exist all the time. Truck impact makes local congestion hard to vanish due to its higher probability to slow down. Traffic flow becomes more congested as the impact of trucks increases. In Figures 5(e)-5(h) the system 


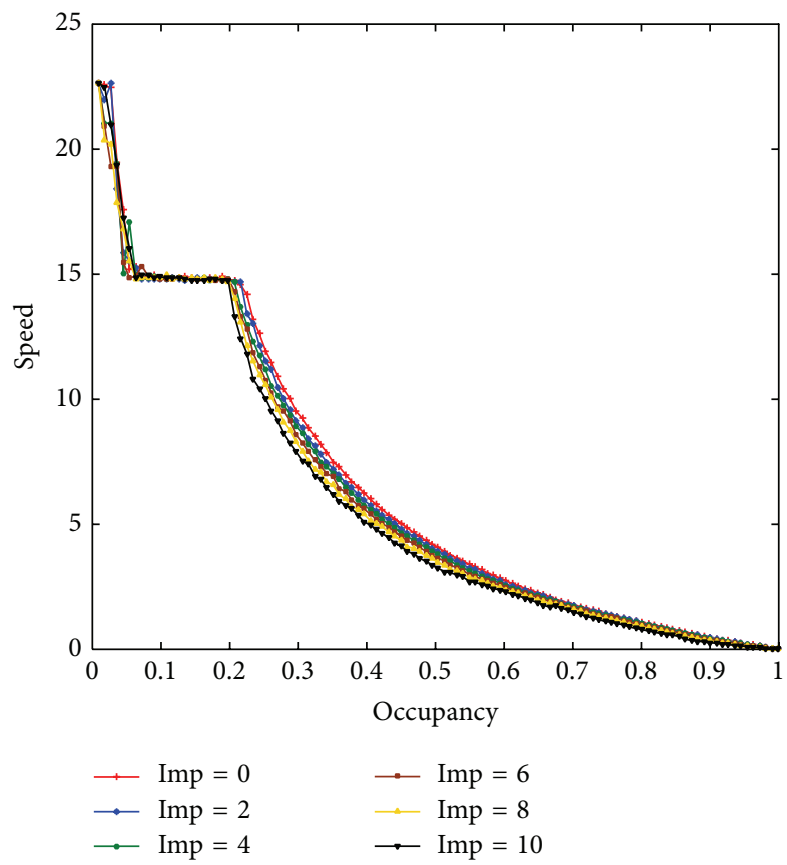

(a) Speed versus occupancy

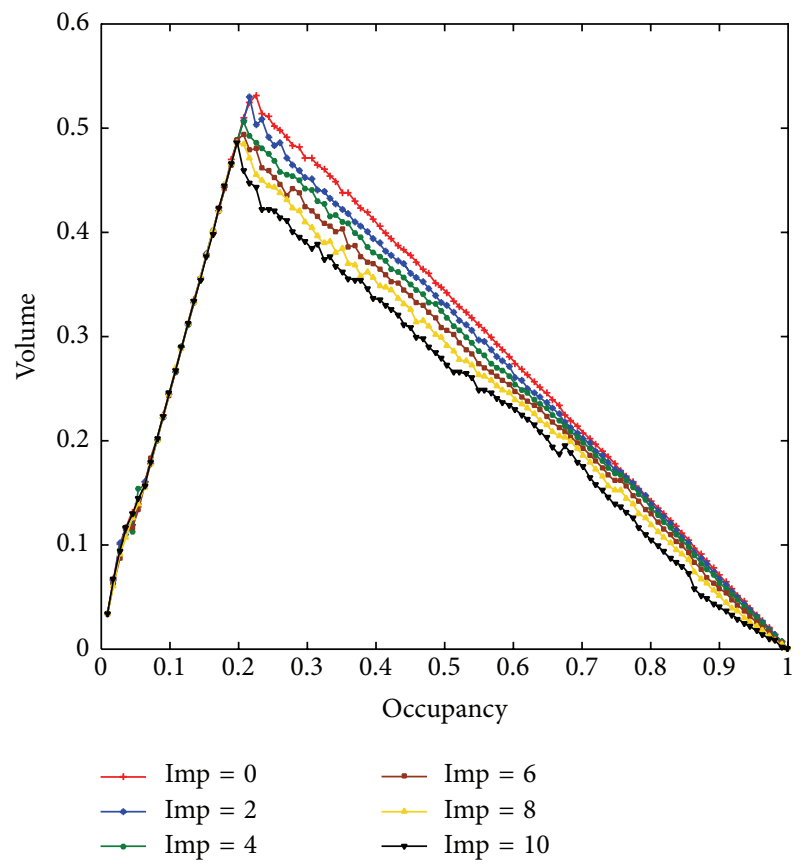

(b) Volume versus occupancy

FIgURE 4: The fundamental diagram under different imp when $r=0.2$.

states are compared at truck percentages of 0 and 0.5 , respectively, when imp $=6$. When the occupancy is 0.05 , vehicles in traffic flow of passenger cars only are evenly distributed with straight tracks, all of which are travelling at maximum speed. In the heterogeneous traffic flow, on the other hand, significant accumulation of vehicles and road vacancy are presented in the figure due to the obstruction by trucks. When the occupancy is 0.225 , traffic flow of passenger cars only already enters the congestion phase and the black areas appear indicating the emergence of traffic congestion in the system. At that time, vehicles in the heterogeneous traffic flow still drive smoothly most of the time despite some small occasional disturbance. This difference also reflects that the critical occupancy of heterogeneous traffic flow is larger than that of traffic flow of passenger cars only.

4.3. Analysis of Speed Variance of Passenger Cars. From the above analysis, the impact of trucks does not have a significant influence on traffic volume and speed when the critical occupancy has not yet been fetched. However, this does not mean absolutely no impact of trucks at that time. The speed variance of passenger cars with different truck impacts when $r=0.2$ was studied and the results are shown in Figure 6. From Figure 6(a), the speed variance of passenger cars increases with the occupancy and reaches the highest value when $C=0.3$. The highest value is among 60.9769.54 by different impacts of trucks. Then it starts to decrease. To better understand the impacts of trucks on the speed variance of passenger cars in different traffic conditions, the relationship between speed variance of passenger cars and the impact of trucks under different occupancies is exhibited in Figures 6(b), 6(c), and 6(d). The impacts of trucks on the speed variance of passenger cars are different under different occupancies and there are three situations. When $C \leq 0.3$, the speed variance of passenger cars changes obviously and increases with the impacts of trucks. For example, the speed variance of passenger cars increases from 30.02 to 40.80 with the growth of the impact of trucks from 0 to 10 at $C=0.1$. When $0.3<C \leq 0.7$, the speed variance of passenger cars changes little. It seems that trucks affect the speed variance of passenger cars without regularity (e.g., the speed variance of passenger cars is almost the same when $C=0.4$, while it decreases slightly when $C=0.5$ ). When $C>0.7$, the speed variance of passenger cars decreases significantly with the impact of trucks. For instance, the speed variance of passenger cars decreases from 5.40 to 1.99 with the growth of the impact of trucks from 0 to 10 when $C=0.9$. Since trucks can affect the lane change of passenger cars in the proposed model, the lane-changing rate of passenger cars has been obtained and shown in Figure 7. The lane-changing rate of passenger cars also increases with the occupancy firstly and reaches the highest value when $C=0.3$ and then decreases. When $C \leq 0.3$, a greater truck impact makes a higher lane-changing rate of passenger cars. Then the lanechanging rate of passenger cars under different truck impacts tends to become close when $C>0.3$. Under the impact of trucks, passenger car drivers decelerate when following the truck and try to change lanes more actively. When there are not many vehicles on the road $(C \leq 0.3)$, there will be many spaces for passenger cars to change lanes. Passenger cars which change lanes successfully can drive at a relatively high speed. However, passenger cars which still drive behind the truck have to drive at a slow speed. Because the average speed of passenger cars does not change, the speed variance 


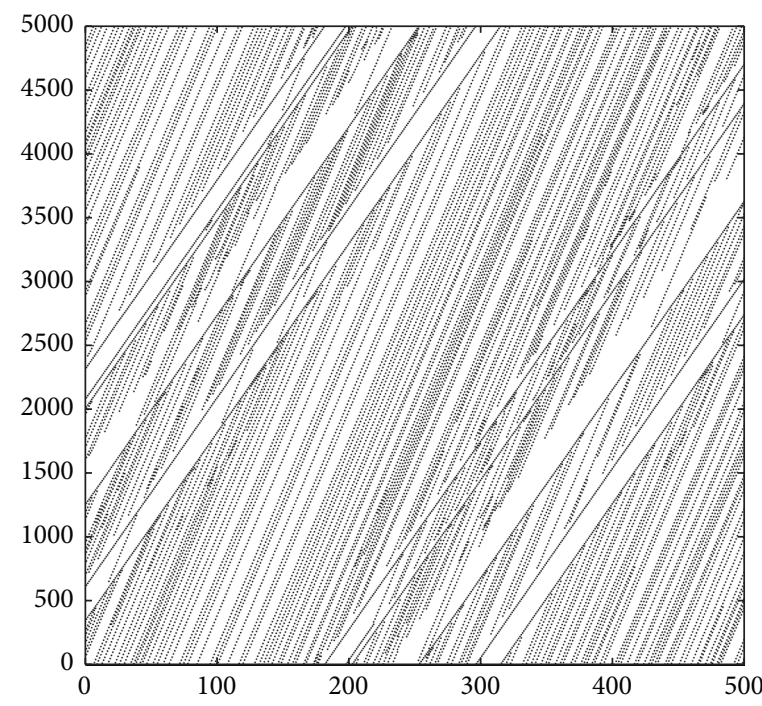

(a) $\operatorname{imp}=0, r=0.2, C=0.05$

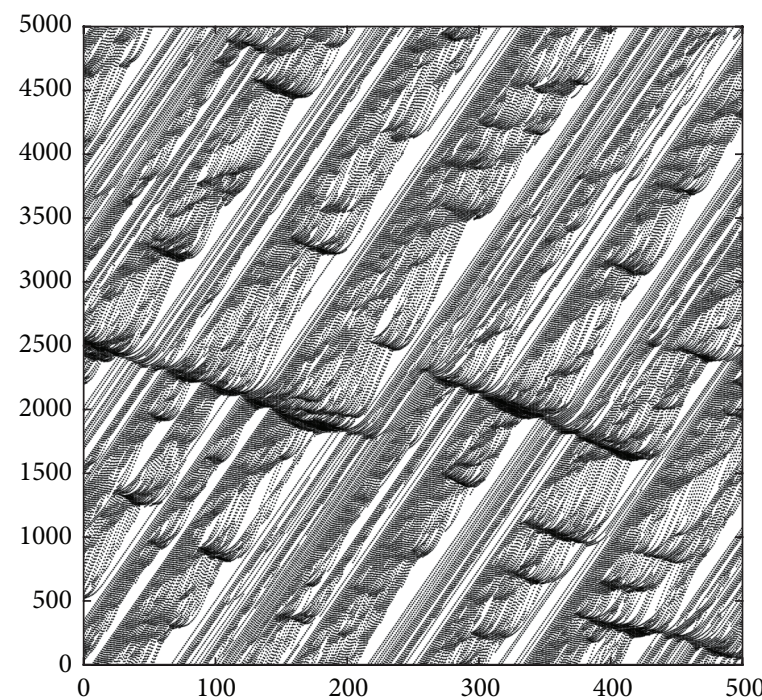

(c) imp $=0, r=0.2, C=0.255$

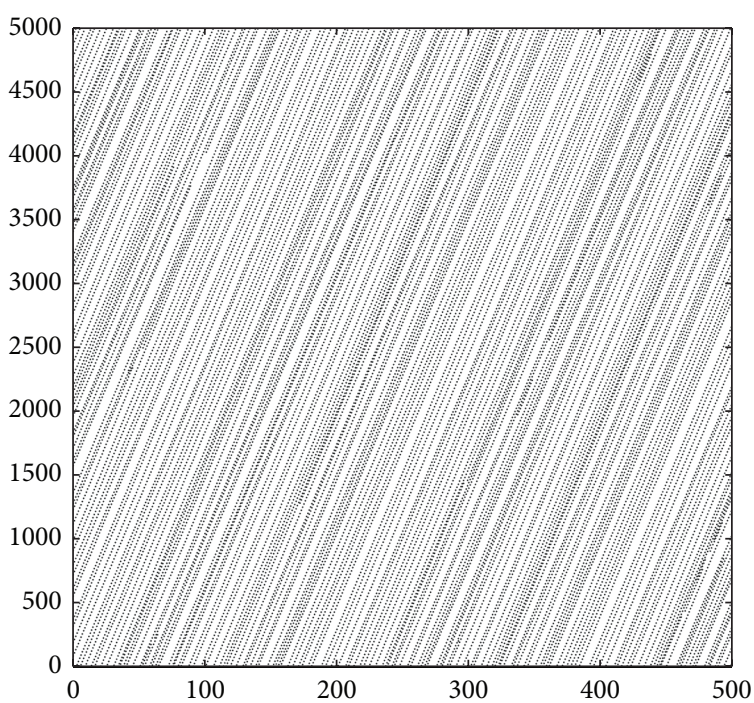

(e) $\operatorname{imp}=6, r=0, C=0.05$

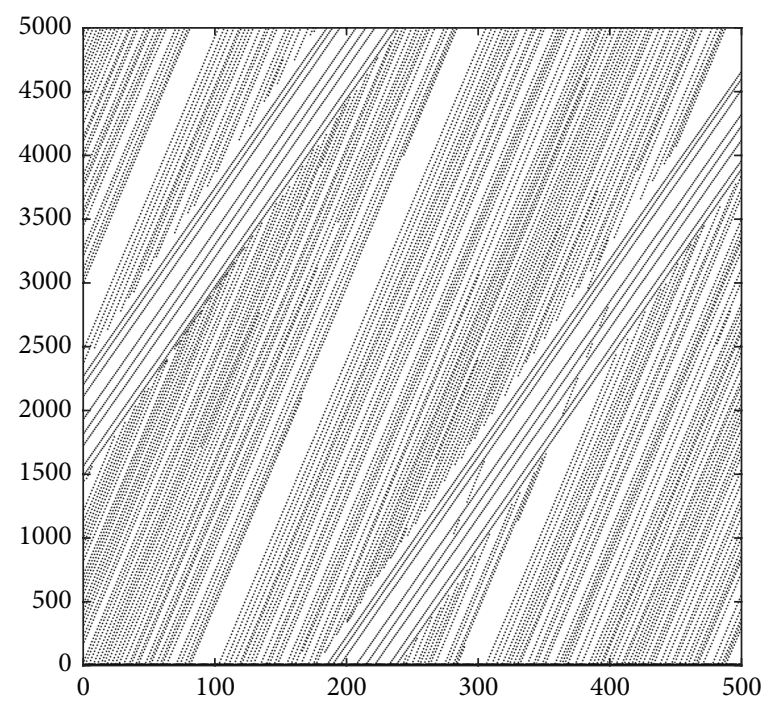

(b) imp $=10, r=0.2, C=0.05$

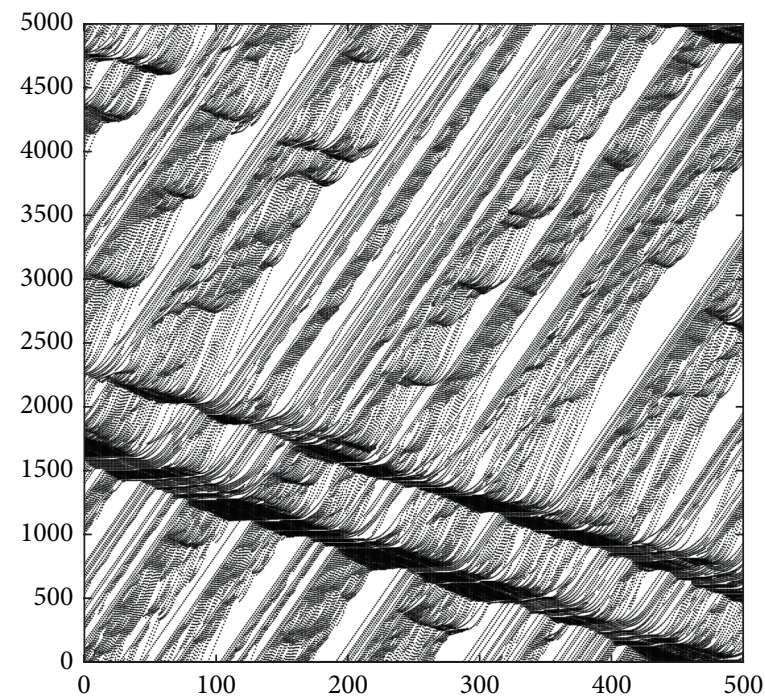

(d) $\mathrm{imp}=10, r=0.2, C=0.255$

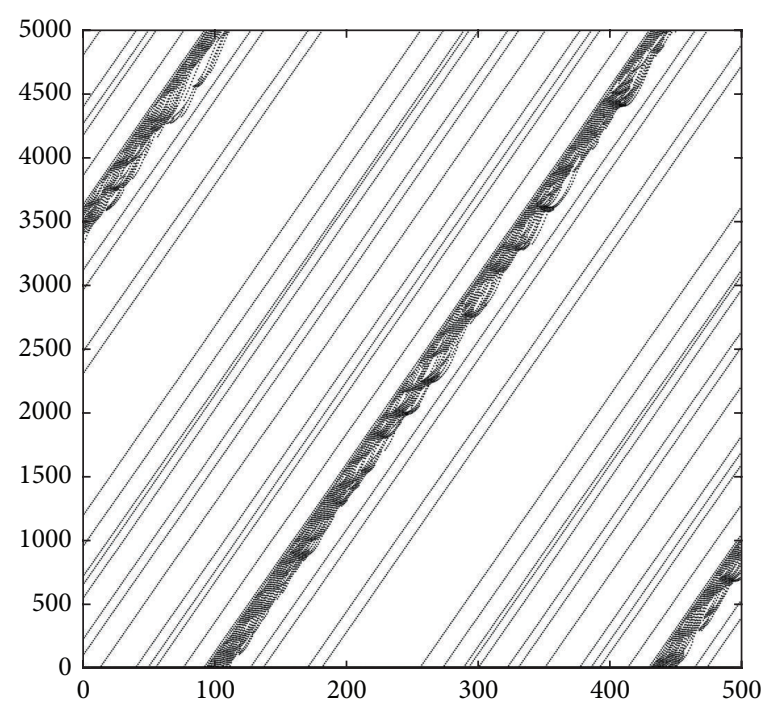

(f) imp $=6, r=0.5, C=0.05$

Figure 5: Continued. 


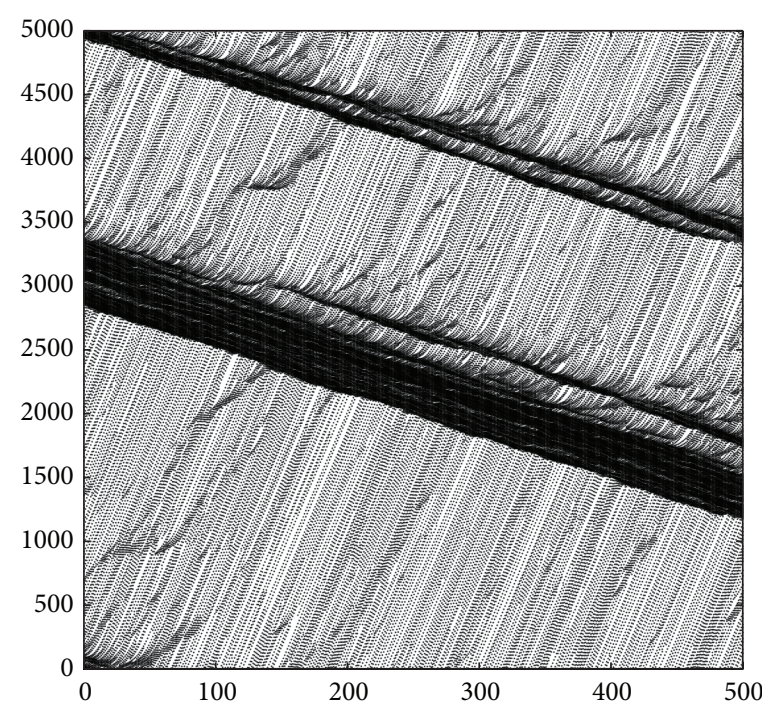

(g) imp $=6, r=0, C=0.255$

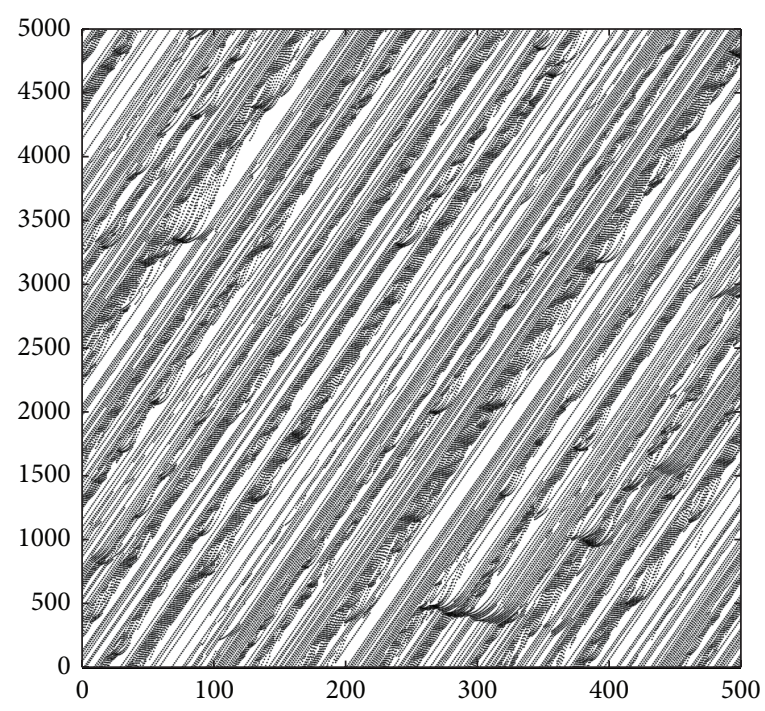

(h) imp $=6, r=0.5, C=0.255$

FIGURE 5: Spatial-temporal diagrams.

becomes large. Truck impact can make this phenomenon more significant so that the speed variance increases with the impact of trucks when $C \leq 0.3$. When there are many vehicles on the road $(C>0.7)$, there are not enough spaces for passenger cars to change lanes. They can only drive behind the truck at slow speed. When the impact of trucks increases, the lane-changing rate remains the same and the speed of passenger car following truck decreases. This effect narrows the speed range of passenger cars and hence reduces their speed variance. The region of $0.3<C \leq 0.7$ is a transition and trucks do not affect the speed variance of passenger cars regularly. Speed variance is an important factor that indicates the stability of a traffic system. From the results, the impact of trucks will deteriorate the stability of traffic flow when the occupancy is low, while it can improve the stability of traffic flow when the occupancy is high.

4.4. Analysis of Space Gap. A space gap is an important parameter in CA models, which determines the choice of deceleration and lane change in the update rules. This section studied the feature of the space gap of car-following-truck (C-T). The relationship between the space gap of C-T and occupancy under different impacts of trucks when $r=0.2$ is presented in Figure 8. Because the average value of space gap is very large and changes a lot in the simulation when there are only a small number of vehicles, we only calculated and analyzed the space gap when $C \geq 0.1$. For ease of comparative analysis, the space gap of car-following-car (CC) was also obtained. When $0.1 \leq C \leq 0.2$, the space gap of C-T is independent of the occupancy. The space gap fluctuates around a certain value. For example, the space gap of C-T is nearly 26.77 when imp $=6$. When $C>0.2$, the space gap of C-T decreases with the occupancy. The impact of trucks has a significant influence on the space gap of CT. Passenger cars will keep a larger space gap if the impact of trucks becomes greater. Comparing the space gap of C-T with that of $\mathrm{C}-\mathrm{C}$, there is an interesting difference between the two kinds of CA models. For the traditional heterogeneous CA model, that is, imp $=0$, the space gap of C-T is smaller than that of $\mathrm{C}-\mathrm{C}$ all the time. For the improved CA model, that is, imp $>0$, the relative relationship between the space gap of C-T and that of C-C is changeable. When imp $\leq 3$ and the occupancy is small, the space gap of C-T is also smaller than that of $\mathrm{C}-\mathrm{C}$. When the occupancy is larger than a certain value, the space gap of $\mathrm{C}$ - $\mathrm{T}$ will become larger than that of C-C. This certain value is related to the impact of trucks. A greater truck impact leads to a smaller value. For example, when imp $=1,2$, and 3 , the certain value is $0.53,0.39$, and 0.26 , respectively. When the impact of trucks is great enough, the space gap of C-T can be always larger than that of C-C (e.g., when imp $\geq 4$ ). These results can be explained by the physical and operational characteristics of vehicles. In the traditional heterogeneous CA model, vehicles are designed to take full advantage of the free cells in front to accelerate to get speed advantages. Since passenger cars have advantages of maximum speed and acceleration compared with trucks, it is easy for passenger cars to catch up and keep a close distance with trucks in the same traffic condition. Hence, the space gap of $\mathrm{C}-\mathrm{T}$ is smaller than that of $\mathrm{C}-\mathrm{C}$ in the traditional heterogeneous CA model. In the improved CA model, although passenger cars still have the advantages of maximum speed and acceleration compared with trucks, passenger car drivers will no longer try to accelerate when following a truck but instead they decelerate in consideration of safety. They will enlarge the space gap between the truck and themselves. Therefore, the space gap of C-T can become larger than that of C-C in the improved CA model. From the questionnaire survey and literatures [5, 26-28], the space gap of C-T should be larger than that of $\mathrm{C}-\mathrm{C}$ in the real world. It indicates that the proposed CA model can reproduce the correct relationship of the space gap between C-T and C-C, but not in the traditional heterogeneous CA model. In the 


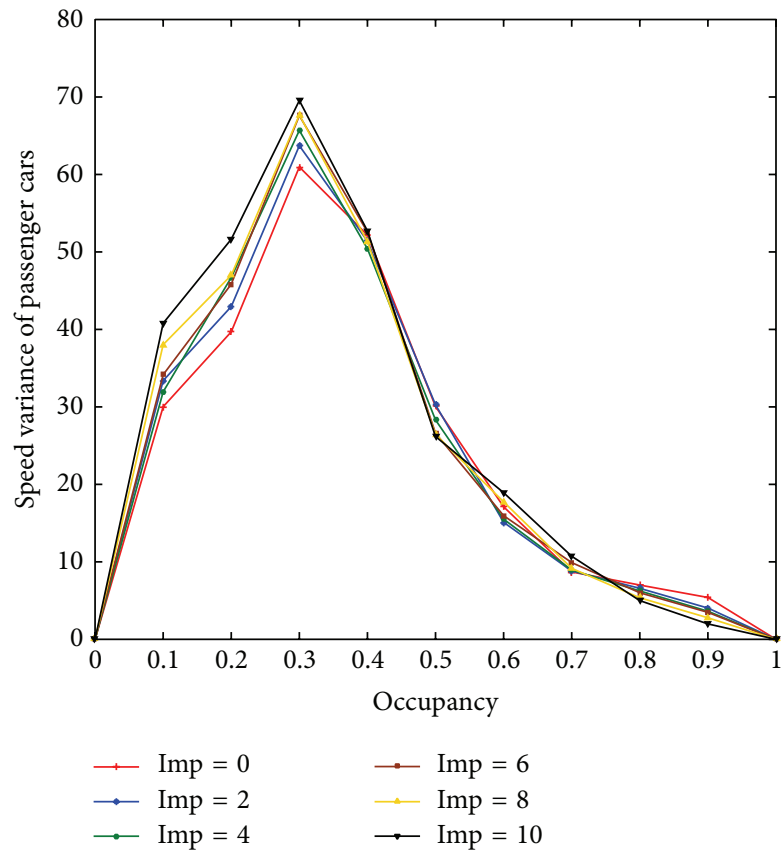

(a) Speed variances of passenger cars

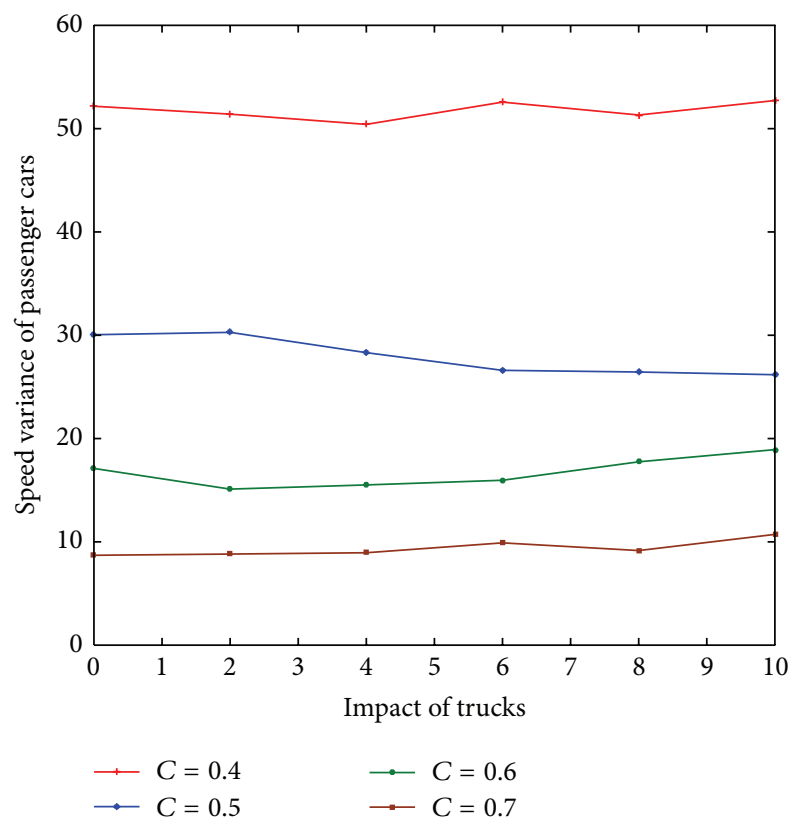

(c) $0.4 \leq \mathrm{C} \leq 0.7$

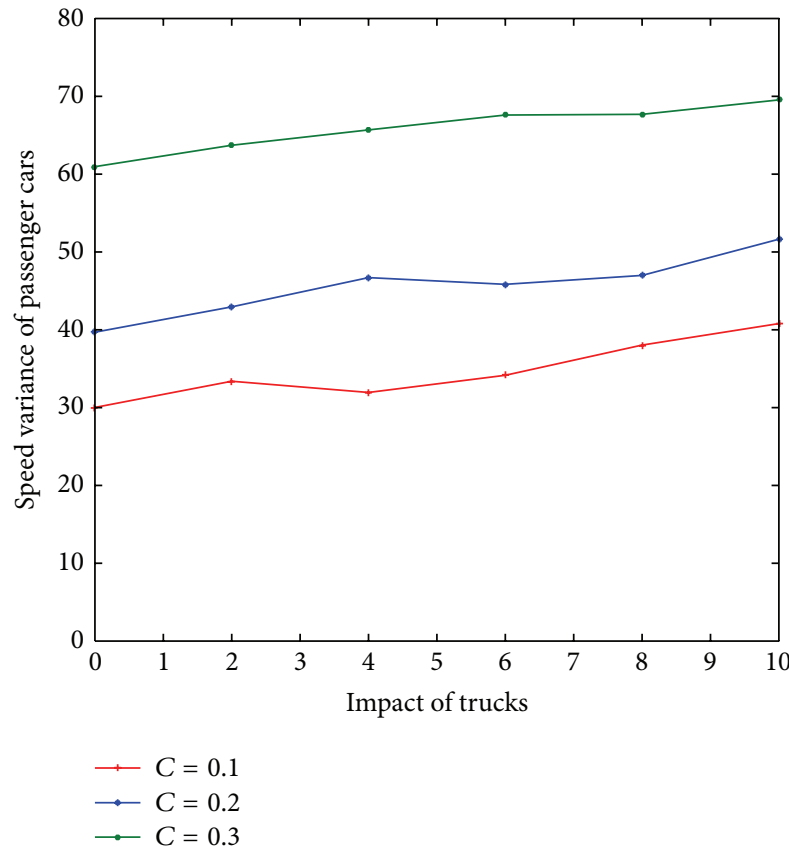

(b) $C \leq 0.3$

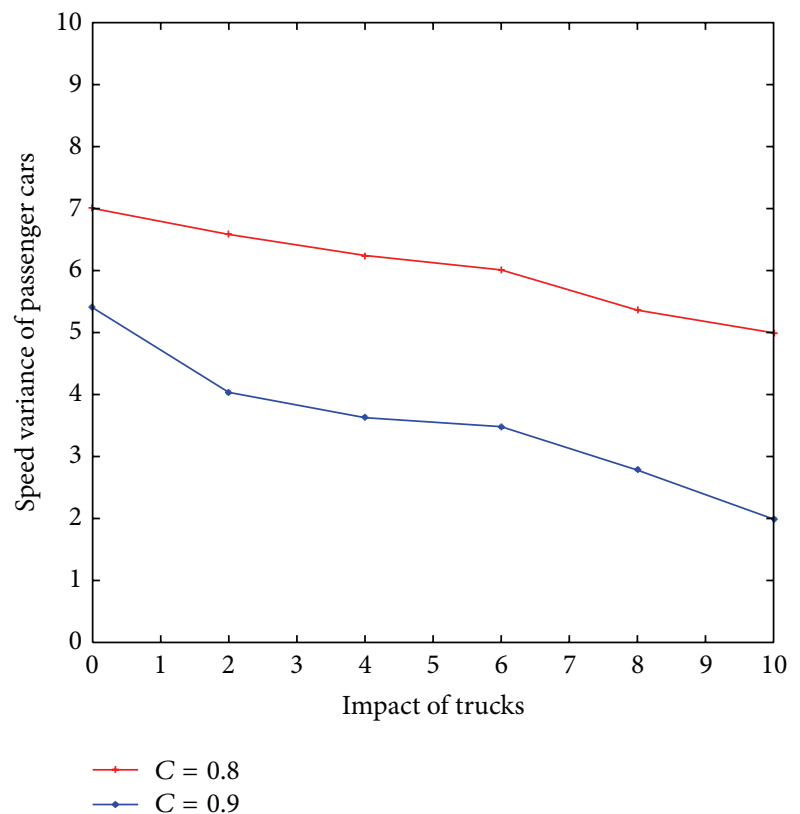

(d) $C \geq 0.8$

FIgURE 6: Speed variances of passenger cars when $r=0.2$.

traditional heterogeneous CA model, the behaviour of the car driver is the same no matter whether cars or trucks are interfered. It means that the truck in the model is not like a truck, but a large-sized passenger car at slow speeds to the passenger car following behind. This difference will affect the analysis of the heterogeneous traffic flow and cause wrong results, which should be paid considerable attention to and improved. For example, space gap between vehicles affects the traffic capacity; if the wrong space gap is obtained, the traffic capacity will also be wrong. To obtain the detailed relationship between the space gap of C-T, occupancy, and truck impact, the linear and quadratic function were used to fit. The curve of space gap of C-T has two regions: in the region $0.1 \leq C<0.2$, the space gap of $\mathrm{C}-\mathrm{T}$ only changes with the impact of trucks; in the region $C>0.2$, both occupancy and truck impact affect the change of the space gap of C-T. The 


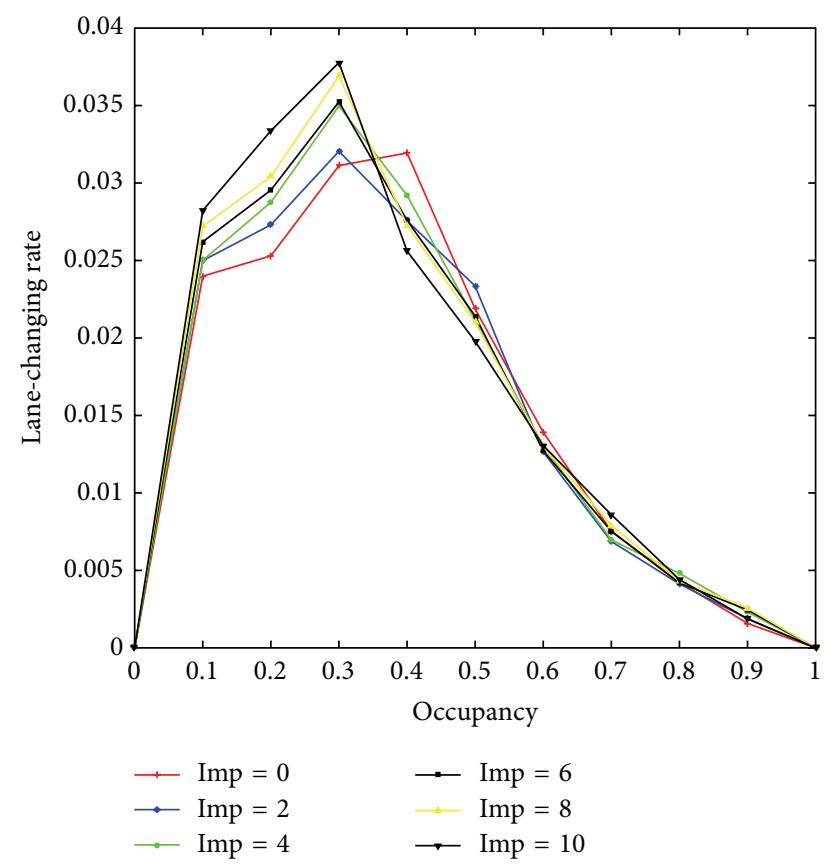

FIGURE 7: Lane-changing rate of passenger cars with different imp when $r=0.2$.

function with the smallest root mean square error (RMSE) and highest adjusted $R^{2}$ will be finally chosen and shown as follows:

$$
\begin{aligned}
\bar{d}_{\mathrm{C}-\mathrm{T}}= & -0.14 * \mathrm{imp}^{2}+2.59 * \mathrm{imp}+16.806, \\
& 0.1 \leq C<0.2,0 \leq \mathrm{imp} \leq 10, \\
\bar{d}_{\mathrm{C}-\mathrm{T}}= & \left(-0.448 * \mathrm{imp}^{2}+2.923 * \mathrm{imp}+24.097\right) * C^{2} \\
& +\left(0.683 * \mathrm{imp}^{2}-6.368 * \mathrm{imp}-48.792\right) \\
& * C-0.218 * \mathrm{imp}^{2}+3.305 * \mathrm{imp} \\
& +25.414, \quad 0.2 \leq C \leq 1,0 \leq \mathrm{imp} \leq 10 .
\end{aligned}
$$

To validate (11), 55 situations were chosen to perform a validation test (each imp selected 5 situations and these 55 situations were not used for equation development). The RMSE and adjusted $R^{2}$ are 0.662 and 0.995 , respectively, which means the equations can explain the space gap of C-T very well. Equations (11) meet the accuracy requirement and can be used to predict the space gap of C-T.

\section{Conclusion and Discussions}

This paper studied the impact of trucks on traffic flow based on the improved CA model. A questionnaire survey was conducted. From the survey, the impact of trucks exists when passenger cars drive behind a truck and it is significant. The behaviour of passenger car drivers changes under the impact of trucks, including enlarging the space, increasing the probability of deceleration, and changing lanes in advance. Grounded upon the existing heterogeneous CA models, the

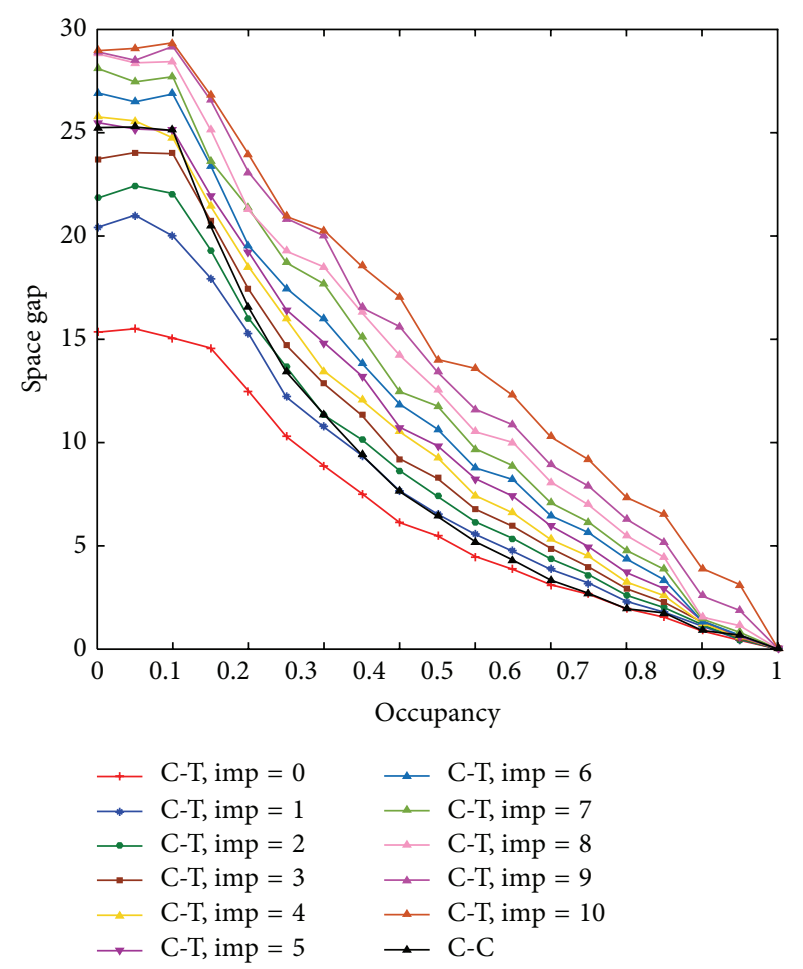

FIGURE 8: The relationship between the space gap of C-T and occupancy under different imp when $r=0.2$.

imp was introduced to reflect the degree to which trucks affect passenger cars quantitatively and the lane-change and car-following rules were modified to build the improved CA model. The simulation results show that (1) compared with the existing heterogeneous CA models, percentages of trucks still have a significant influence on traffic volume in the congestion phase in the proposed model and high mixed degree can cause a greater decline in traffic volumes. Traffic volume and speed decrease with the impact of trucks only in the congestion phase. (2) When the occupancy is high, truck impact makes the local congestion hard to vanish and traffic flow becomes more congested as the impact of trucks increases. (3) The speed variance of passenger cars is proportional to the impact of trucks when the occupancy is low and it becomes inversely proportional to the impact of trucks when the occupancy is high. (4) The space gap of C-T is affected by both occupancy and the impact of trucks. The space gap of C-T is not always smaller than that of $\mathrm{C}-\mathrm{C}$ in the proposed model. The relative relationship is changeable at a certain value of occupancy, which is related to the impact of trucks.

The proposed CA model has considered not only the performance differences between passenger cars and trucks, but also the impact of trucks on the behaviour of passenger cars. It can reflect the impact of trucks on traffic flow more comprehensively and improve the accuracy of the simulation of traffic flow. The simulation results can help to understand the impact of trucks on traffic flow further and provide a theoretical basis for improving road traffic efficiency and safety. 
With limited resources and time, there are still some aspects this study could improve. Firstly, this study does not determine the magnitude of the impact of trucks specifically and uses the identical value. It is important to understand how the magnitude of the impact of trucks changes in different conditions by different factors. This could help to improve the accuracy of simulation in the further study and we will collect more field data to study this aspect. For the convenience of analysis, this paper only used the linear and quadratic functions to fit the space gap of $\mathrm{C}-\mathrm{T}$. The accuracy will be further improved if more function forms are used. Also, the impacts of trucks on trucks exist but are not as obvious as on passenger cars. This part is not explored in this study and can be considered further. Meanwhile, the responses of different kinds of vehicle drivers to the impact of trucks will be a focus in the next study to further improve the correctness and applicability of the proposed model.

\section{Competing Interests}

The authors declare that they have no competing interests.

\section{Acknowledgments}

This research is funded by the Young Scientists Fund of the National Natural Science Foundation of China (no. 51408253) and the Jiangsu Traffic Scientific Research Project (no. 2011Y-30).

\section{References}

[1] L. Duan, X. Guo, and C. Yao, "Characteristics of traffic flow on 8lane freeway under lane restriction strategy," Journal of Highway and Transportation Research and Development, vol. 31, no. 4, pp. 125-129, 2014.

[2] Bureau of Transportation Statistics, National Transportation Statistics, Bureau of Transportation Statistics, Washington, DC, USA, 2002.

[3] Bureau of Transport and Regional Economics, Urban Pollutant Emissions from Motor Vehicles: Australian Trends to 2020, Bureau of Transport and Regional Economics, 2003.

[4] M. Sarvi, "Heavy commercial vehicles-following behavior and interactions with different vehicle classes," Journal of Advanced Transportation, vol. 47, no. 6, pp. 572-580, 2013.

[5] D. Kong and X. Guo, "Analysis of vehicle headway distribution on multi-lane freeway considering car-truck interaction," Advances in Mechanical Engineering, vol. 8, no. 4, pp. 1-12, 2016.

[6] M. Bando, K. Hasebe, A. Nakayama, A. Shibata, and Y. Sugiyama, "Dynamical model of traffic congestion and numerical simulation," Physical Review E, vol. 51, no. 2, pp. 1035-1042, 1995.

[7] T. Nagatani, “The physics of traffic jams," Reports on Progress in Physics, vol. 65, no. 9, pp. 1331-1386, 2002.

[8] S. Di Gregorio and D. C. Festa, "Cellular automata for freeway traffic," in Proceedings of the 1st International Conference "Applied Modelling and Simulation", vol. V, pp. 133-136, Lyon, France, September 1981.

[9] M. Cremer and J. Ludwig, "A fast simulation-model for traffic flow on the basis of Boolean operations," Mathematics and Computers in Simulation, vol. 28, no. 4, pp. 297-303, 1986.
[10] K. Nagel and M. Schreckenberg, "A cellular automaton model for freeway traffic," Journal de Physique I, vol. 2, no. 12, pp. 22212229, 1992.

[11] J. Tian, M. Treiber, S. Ma, B. Jia, and W. Zhang, "Microscopic driving theory with oscillatory congested states: model and empirical verification," Transportation Research Part B: Methodological, vol. 71, pp. 138-157, 2015.

[12] P. Chakroborty and A. K. Maurya, "Microscopic analysis of cellular automata based traffic flow models and an improved model," Transport Reviews, vol. 28, no. 6, pp. 717-734, 2008.

[13] M. Fukui and Y. Ishibashi, "Traffic flow in ID cellular automaton model including cars moving with high speed," Journal of the Physical Society of Japan, vol. 65, no. 6, pp. 1868-1870, 1996.

[14] R. Barlovic, L. Santen, A. Schadschneider, and M. Schreckenberg, "Metastable states in cellular automata for traffic flow," The European Physical Journal B-Condensed Matter and Complex Systems, vol. 5, no. 3, pp. 793-800, 1998.

[15] W. Knospe, L. Santen, A. Schadschneider, and M. Schreckenberg, "Towards a realistic microscopic description of highway traffic," Journal of Physics A: Mathematical and General, vol. 33, no. 48, pp. L477-L485, 2000.

[16] M. Jing, W. Deng, H. Wang, and Y.-J. Ji, "Two-lane cellular automaton traffic model based on car following behavior," Acta Physica Sinica, vol. 61, no. 24, Article ID 244502, 2012.

[17] B. Jia, X.-G. Li, R. Jiang, and Z.-Y. Gao, "Traffic behavior around the weaving section in cellular automata model," International Journal of Modern Physics C, vol. 21, no. 3, pp. 409-422, 2010.

[18] H. Ding, W. Wang, T. Luo, Z. Yang, Y. Li, and Z. Li, "Cellular automata based modeling for evaluating different bus stop designs in China," Discrete Dynamics in Nature and Society, vol. 2015, Article ID 365412, 10 pages, 2015.

[19] Q. Meng and J. Weng, "An improved cellular automata model for heterogeneous work zone traffic," Transportation Research Part C: Emerging Technologies, vol. 19, no. 6, pp. 1263-1275, 2011.

[20] B. Jia, R. Jiang, Z. I.-Y. Gao, and X.-M. Zhao, "The effect of mixed vehicles on traffic flow in two lane cellular automata model," International Journal of Modern Physics C, vol. 16, no. 10, pp. 1617-1627, 2005.

[21] A. Ebersbach, J. Schneider, I. Morgenstern, and R. Hammwöhner, "The influence of trucks on traffic flowan investigation on the Nagel-Schreckenberg-model," International Journal of Modern Physics C, vol. 11, no. 4, pp. 837-842, 2000.

[22] R. Xiao, L. Kong, and M. Liu, "The influence of the length and the velocity of vehicles on the mixed traffic flow in one-lane highway," Acta Physica Sinica, vol. 56, no. 2, pp. 740-747, 2007.

[23] S. Moridpour, E. Mazloumi, and M. Mesbah, "Impact of heavy vehicles on surrounding traffic characteristics," Journal of Advanced Transportation, vol. 49, no. 4, pp. 535-552, 2015.

[24] G. H. Bham and R. F. Benekohal, "A high fidelity traffic simulation model based on cellular automata and car-following concepts," Transportation Research Part C: Emerging Technologies, vol. 12, no. 1, pp. 1-32, 2004.

[25] R.-S. Zheng, H.-L. Tan, L.-J. Kong, and M.-R. Liu, "Cellular automaton model for mixing traffic in two-lane system," Acta Physica Sinica, vol. 54, no. 8, pp. 3516-3522, 2005.

[26] K. Aghabayk, M. Sarvi, and W. Young, "Understanding the dynamics of heavy vehicle interactions in car-following," Journal of Transportation Engineering, vol. 138, no. 12, pp. 1468-1475, 2012. 
[27] J. Weng, Q. Meng, and T. F. Fwa, "Vehicle headway distribution in work zones," Transportmetrica A: Transport Science, vol. 10, no. 4, pp. 285-303, 2014.

[28] S. Peeta, P. Zhang, and W. Zhou, "Behavior-based analysis of freeway car-truck interactions and related mitigation strategies," Transportation Research Part B: Methodological, vol. 39, no. 5, pp. 417-451, 2005. 


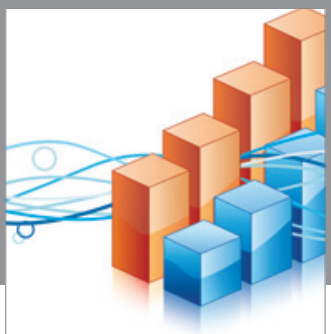

Advances in

Operations Research

vatem alat4

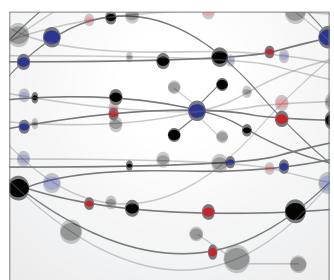

\section{The Scientific} World Journal
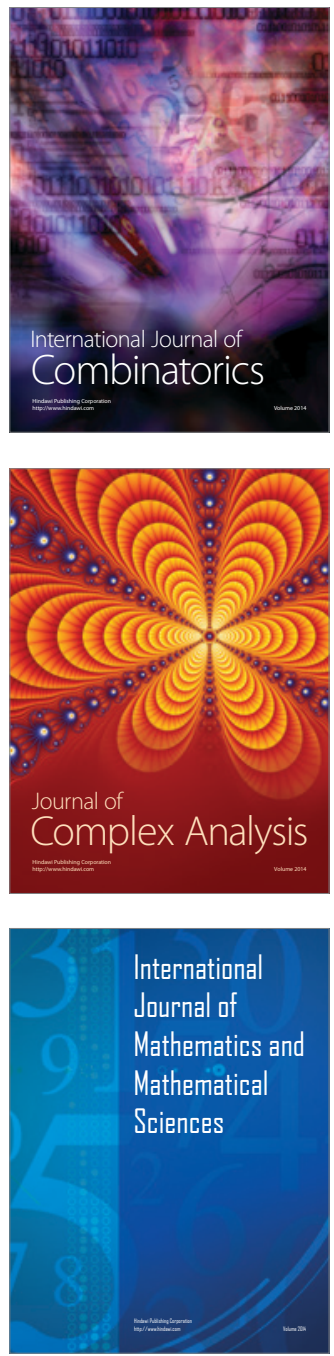
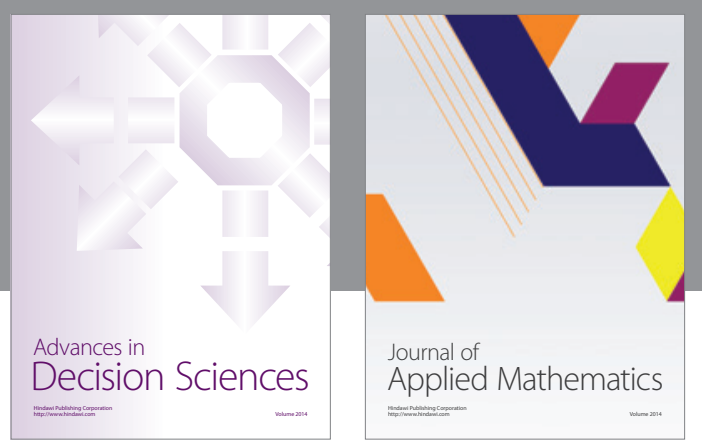

Algebra

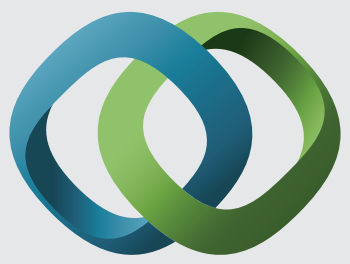

\section{Hindawi}

Submit your manuscripts at

http://www.hindawi.com
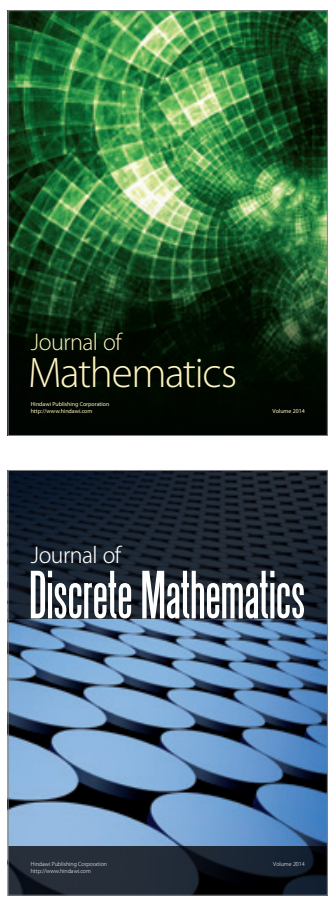

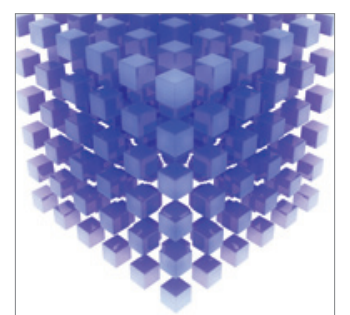

Mathematical Problems in Engineering
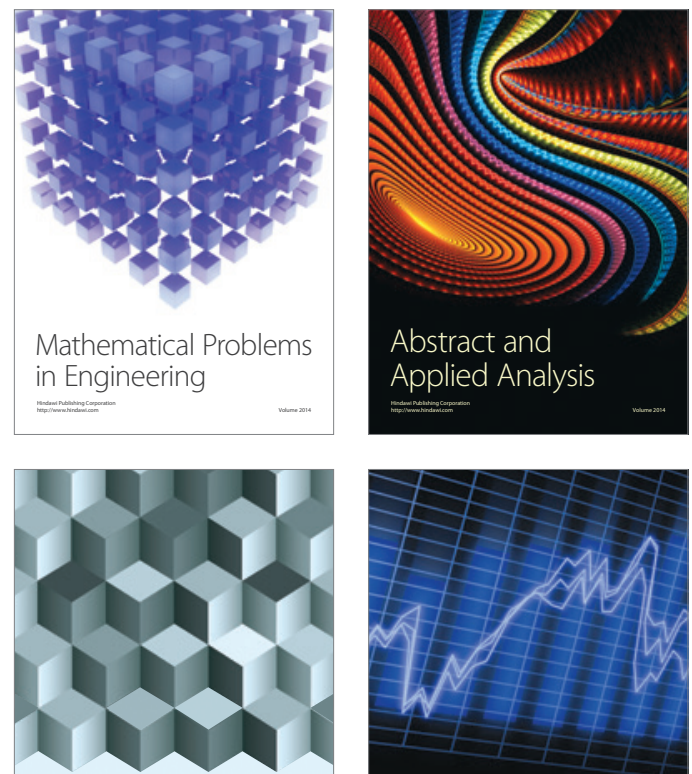

Journal of

Function Spaces

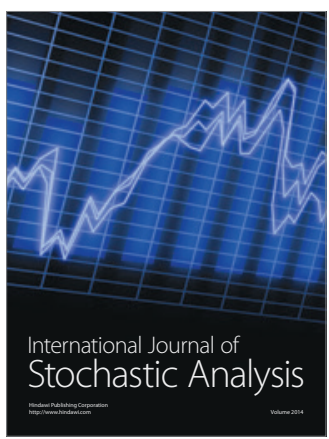

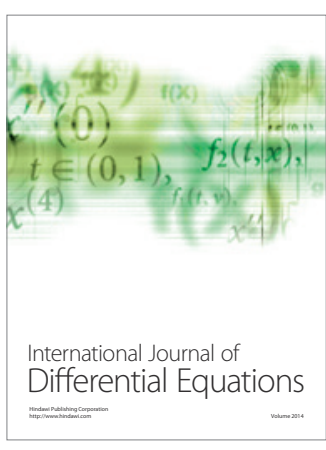
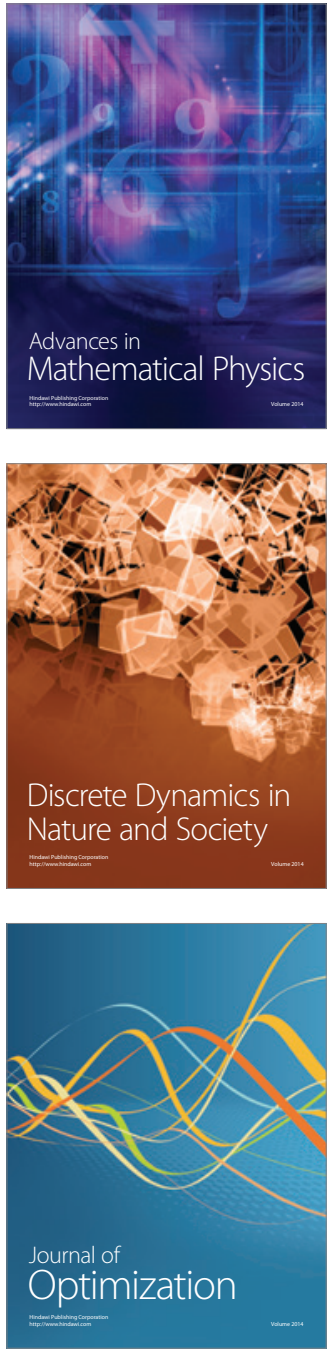